\title{
Lectura y rendimiento académico. Actuaciones desde la biblioteca universitaria
}

\section{Reading and academic achievement. Actions from the academic library}

\section{Sandra Sánchez-García}

Sánchez-García, Sandra (2019). Lectura y rendimiento académico. Actuaciones desde la biblioteca universitaria". Anuario ThinkEPI, v. 13, e13b03.

https://doi.org/10.3145/thinkepi.2019.e13b03

Publicado en IweTel el 3 de julio de 2019

\section{Sandra Sánchez-García}

https://orcid.org/0000-0002-7157-1826

Universidad de Castilla-La Mancha

Grupo PEL (Psicología, Educación y Lectura)

Avda. de los Alfares, 42. 16071 Cuenca

sandra.sanchez@uclm.es

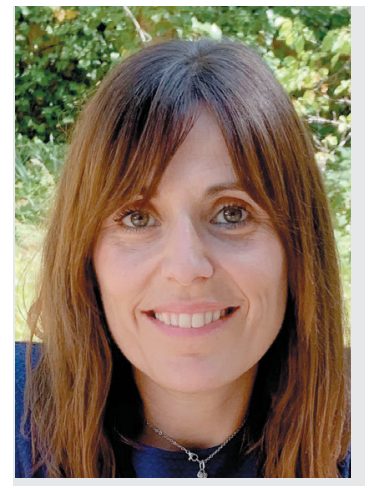

Resumen: En los últimos años se ha potenciado el interés de la investigación en torno a las variables que determinan el éxito académico en las etapas educativas. Muchas investigaciones sitúan a la lectura y al hábito lector como una de las variables que en mayor medida favorecen los resultados académicos. Este hecho contrasta con los datos sobre hábitos de lectura en España, siendo preocupante el porcentaje de jóvenes y alumnos universitarios que no muestran ningún interés por la lectura. En este trabajo se presenta la importancia de promocionar la lectura en todos los niveles educativos, especialmente en la universidad.

Palabras clave: Hábitos de lectura; Motivación lectora; Rendimiento académico; Estudiantes universitarios; Bibliotecas universitarias; Universidad; Promoción lectora.

Abstract: In recent years research has been devoted to the variables that determine academic achievement within the different educational levels. Many researches place reading and reading habit as one of the variables that most favor academic results. This fact contrasts with the data on reading habits in Spain, being worrisome the percentage of young people and college students who show no interest in reading. In this paper we discuss the importance of promoting reading at all educational levels, especially in the university context.

Keywords: Reading habits; Reading motivation; Academic achievement; College students; Academic libraries; Universidad; Reading promotion.

\section{Lectura y rendimiento académico}

Las investigaciones en torno a las variables que determinan el éxito académico han sido una constante en los estudios de educación, evidenciándose, en los últimos años, un interés creciente de las investigaciones que centran su atención en el papel de la lectura y su vinculación con el rendimiento académico (Lluch; Sanchez-García, 2017). Lo que
"La lectura es una de las actividades cotidianas de las personas en formación, entendiéndose como una competencia básica y transversal que permite el acceso al resto de los aprendizajes" 


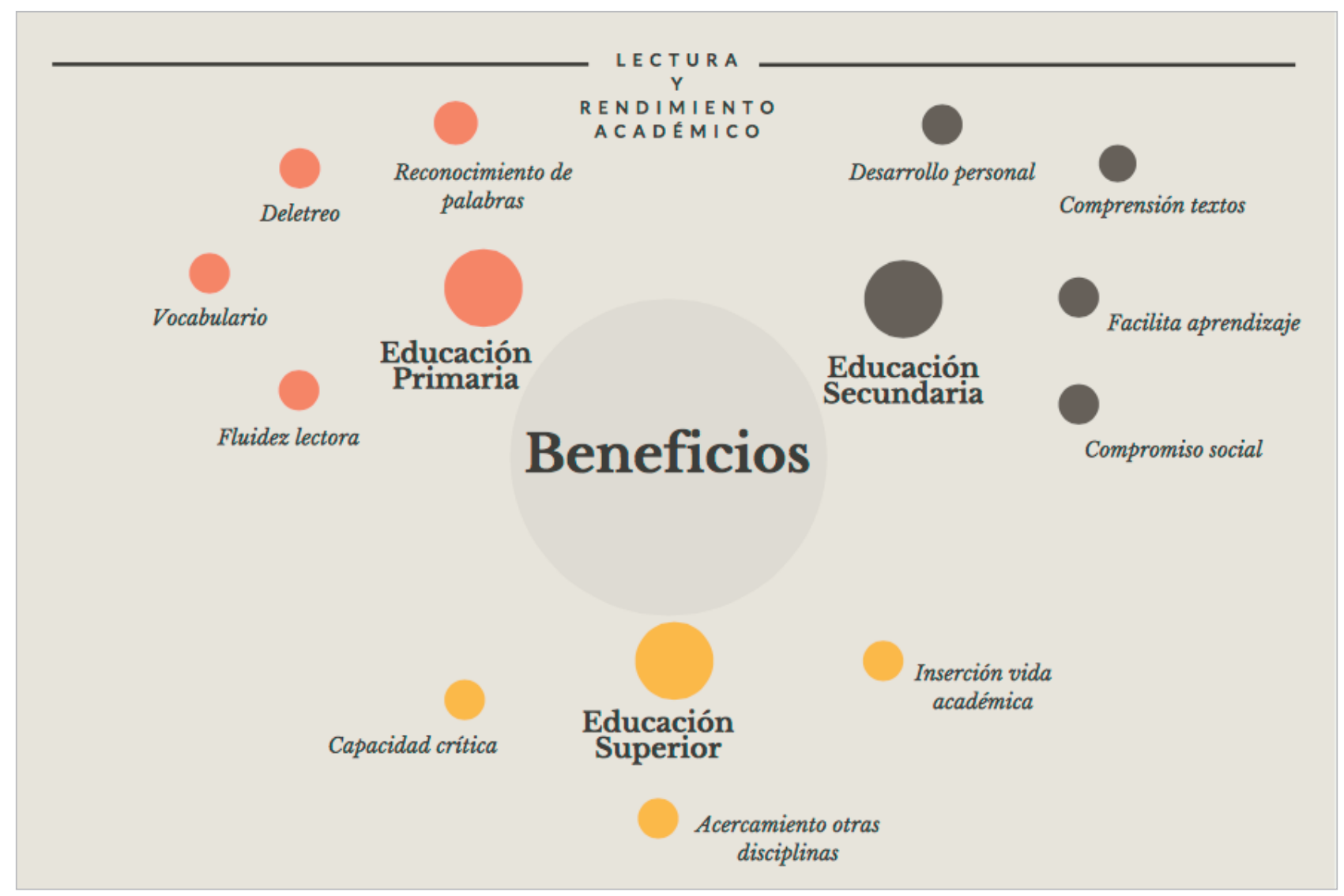

Beneficios de la lectura

resulta totalmente comprensible si tenemos en cuenta que la lectura es una de las actividades cotidianas de las personas en formación, entendiéndose como una competencia básica y transversal que permite el acceso al resto de los aprendizajes. Estos trabajos evidencian en primer lugar cómo la competencia lectora correlaciona en un alto grado con el nivel de consecución de otras competencias genéricas y conocimiento específicos, incidiendo de forma directa en los resultados de los estudiantes, presentando diferentes enfoques según la etapa educativa a la que se refieren.

En las investigaciones centradas en los escolares se han encontrado relaciones significativas entre sus calificaciones y su capacidad de comprensión lectora (Fiz et al., 2000; Pascual-Gómez; Carril-Martínez, 2017, González; Delgado, 2009); presentando evidencias de su relación con el rendimiento en todas las áreas curriculares. En Educación Primaria se destaca, especialmente, la relación positiva entre la lectura por placer y los resultados académicos (Dezcallar et al., 2014; Cunningham; Stanovich, 1991; Ritchie; Bates, 2013). Estas investigaciones confirman cómo la cantidad de tiempo dedicado a la lectura recreativa fuera de la escuela favorece la obtención de mejores puntuaciones en deletreo, vocabulario, reconocimiento de palabras y fluidez verbal.

En los trabajos referidos a los estudiantes adolescentes se observa una relación directa entre su rendimiento académico y el hábito lector, entendido también como placer por la lectura y lectura voluntaria (Molina-Villaseñor, 2006; Mol; Jolles, 2014, Gil-Flores, 2011; Gambrell, 2015; Howard, 2011; Hughes-Hassell; Rodge, 2007), mejorando su comprensión lectora y favoreciendo los aprendizajes en todas las disciplinas. No olvidemos que los informes PISA ya concluían que los jóvenes que leen diariamente por placer obtienen mejores resultados en estas pruebas. En Educación Secundaria Obligatoria está ampliamente documentada la existencia de una relación positiva entre la lectura con propósito recreativo y los resultados académicos, además de otras competencias adquiridas por los jóvenes que relacionan este hábito con un mayor compromiso social y desarrollo personal. 
En el ámbito universitario como señalan Moyano (2014) y Carlino, (2013), también tienen mejor desarrollo en su disciplina y una mejor inserción en la vida académica los estudiantes que se declaran lectores. El hábito lector es por tanto uno de los factores que en mayor medida se relaciona con la obtención de mejores calificaciones también en la educación superior, siendo la lectura voluntaria y recreativa la que presenta una correlación directa con el desarrollo de hábitos de lectura estables (Elche; Sánchez-García; Yubero, 2019; Fraguela-Vale; Pose-Porto; Varela-Garrote, 2016; Lee, 2014).

En todos estos estudios podemos observar cómo adquiere gran relevancia la lectura recreativa o voluntaria, la que se realiza libremente durante el tiempo libre y de ocio, al margen de las actividades académicas y por propia decisión y libertad de elección de material, ya que es precisamente este tipo de lecturas las que definen a las personas como lectoras. Por lo tanto, parece imprescindible fomentar el placer por la lectura fuera del ámbito propiamente académico. Leer por placer es una herramienta efectiva y debería estar al alcance de todos los alumnos independientemente de la etapa educativa en la que se encuentre.

“Es imprescindible no centrar sólo el interés de los alumnos por la lectura con un valor instrumental y se ha de favorecer también desde la institución el comportamiento lector voluntario, ya que es generador fundamental de los hábitos lectores" (Yubero; Larrañaga, 2015, p. 719).

\section{Los hábitos de lectura de los estudiantes universitarios}

En este sentido debemos de ser conscientes de que el porcentaje de jóvenes que seleccionan la lectura como una actividad de ocio es reducido. A partir de los 14 años su interés por la lectura desciende notablemente, valorándola en muchos casos sólo por su carácter instrumental. Según el estudio realizado por la Fundación SM (González-Anleo; López-Ruiz, 2017), casi el 32\% de los jóvenes españoles declara no haber leído ni un solo libro durante 2016. Esto, unido al casi $40 \%$ que leen solamente entre 1-3 libros, hace que nos encontremos ante un dato ciertamente alarmante si tenemos en cuenta que la mayor parte de los entrevistados son estudiantes y el estudio no diferencia entre lectura obligatoria y lectura voluntaria. Estos datos refuerzan las aportaciones de numerosos informes realizados en los últimos años por el CIS (Centro de Investigaciones Sociológicas) y la FGEE (Federación de Gremio de Editores de España) que informan sobre esta realidad en nuestro país.

Esto mismo sucede con los jóvenes universitarios.

Autores como Gilbert y Fister (2011), Elliot (2009), Gladwin y Goulding (2012), Yubero y Larrañaga (2015) y Fraguela-Vale, Pose-Porto y Varela-Garrote (2016), ofrecen datos cuantitativos sobre los hábitos y las tendencias lectoras de la población universitaria, evidenciando la necesidad de no descuidar la formación del hábito lector y el desarrollo

“Un $\mathbf{8 7 , 8 \%}$ de jóvenes declara no leer nunca como actividad de ocio, lo que evidencia el hecho de que muchos universitarios no tienen insertada la lectura en su estilo de vida" de la competencia lectora entre los universitarios.

Como señalan Yubero y Larrañaga (2015), casi el 16\% de los estudiantes universitarios españoles no lee nunca, casi un $8 \%$ no ha leído ningún libro en el último año y el $25 \%$ lee algún libro solo de forma ocasional. Si centramos los datos a la lectura voluntaria, Fraguela-Vale, Pose-Porto y Varela-Garrote (2016), señalan que un $87,8 \%$ de jóvenes declara no leer nunca como actividad de ocio, lo que evidencia el hecho de que muchos universitarios no tienen insertada la lectura en su estilo de vida.

Los trabajos de Larrañaga, Yubero y Cerrillo (2008), Larrañaga y Yubero (2005) y Yubero y Larrañaga (2015), informan, además, de una serie de inconsistencias o contradicciones que aparecen en el cruce de varias respuestas, lo que llevó a los autores a identificar la categoría de "falsos lectores" para referirse a aquellos estudiantes que, aún valorando de forma positiva la lectura, esta no forma parte de su estilo de vida. Estos estudiantes dan una imagen falsa de lector, ya que por su frecuencia e intensidad lectora no se les puede considerar como tales. Estos estudiantes pueden falsear sus respuestas en encuestas sobre hábitos de lectura, en función de la deseabilidad social y utilizando el autoengaño.

Estas investigaciones, además, confirman cómo buena parte del índice lector de los estudiantes universitarios españoles se refiere a una lectura instrumental.

Ante estos datos, y siendo conscientes de la relación existente entre hábito lector, comprensión lectora y rendimiento académico, también en el ámbito universitario es preciso favorecer el comportamiento lector voluntario, ya que es el generador

"Solemos pensar, erróneamente, que los alumnos universitarios poseen hábito lector y tienen las competencias lectoras necesarias para desarrollar sus estudios con eficacia" 
"Resulta contradictorio que entre los objetivos de la educación primaria se incluya el desarrollo del gusto por la lectura y que en las siguientes etapas educativas (obligatorias y no obligatorias) desaparezca este aspecto por completo, quedando la lectura relegada exclusivamente a su valor instrumental y obligatorio" (Yubero; Larrañaga, 2015, p. 719).

Como señalan estos autores, solemos pensar, erróneamente, que los alumnos universitarios poseen hábito lector y tienen las competencias lectoras necesarias para desarrollar sus estudios con eficacia, hecho que resulta al menos paradójico ante los datos expuestos.

\section{Bibliotecas universitarias y promoción lectora}

Ante esta situación entendemos que es necesario proporcionar espacios de lectura que potencien el comportamiento lector de los universitarios y apoyen el desarrollo y la mejora de sus hábitos lectores. La universidad y las bibliotecas universitarias deben implicarse y proporcionar espacios y programas de promoción lectora para los estudiantes universitarios.

Muchas universidades ofrecen a sus estudiantes opciones para el tiempo libre poniendo a su disposición actividades, equipamientos e infraestructuras como complemento de la formación académica. Dentro de esta oferta, integrados en los programas de actividades culturales o, incluso, de competencias transversales, la universidad debería generar espacios de lectura dinámicos, flexibles y participativos que contribuyan al desarrollo del hábito lector. En este contexto, las bibliotecas universitarias se convierten en el agente más idóneo para llevar a cabo programas de promoción lectora que ofrezcan a estos alumnos colecciones de ocio, espacios para la lectura y actividades en los que se fomente el diálogo y el debate en torno a lecturas no académicas (Sánchez-García; Yubero, 2015; 2017).

Son muchas las universidades españolas sensibles a esta necesidad. Muestra de ello es la creación en 2007 de la Red Internacional de Universidades Lectoras, formada por 46 universidades de España, Portugal, Italia y Latinoamérica, con el objetivo de intentar contrarrestar las carencias en lectura que presentan los universitarios. Las iniciativas llevadas a cabo de forma cooperativa por esta red pretenden potenciar el hábito de la lectura y la escritura entre los universitarios, no sólo como herramienta de trabajo, sino sobre todo como vehículo de promoción integral.

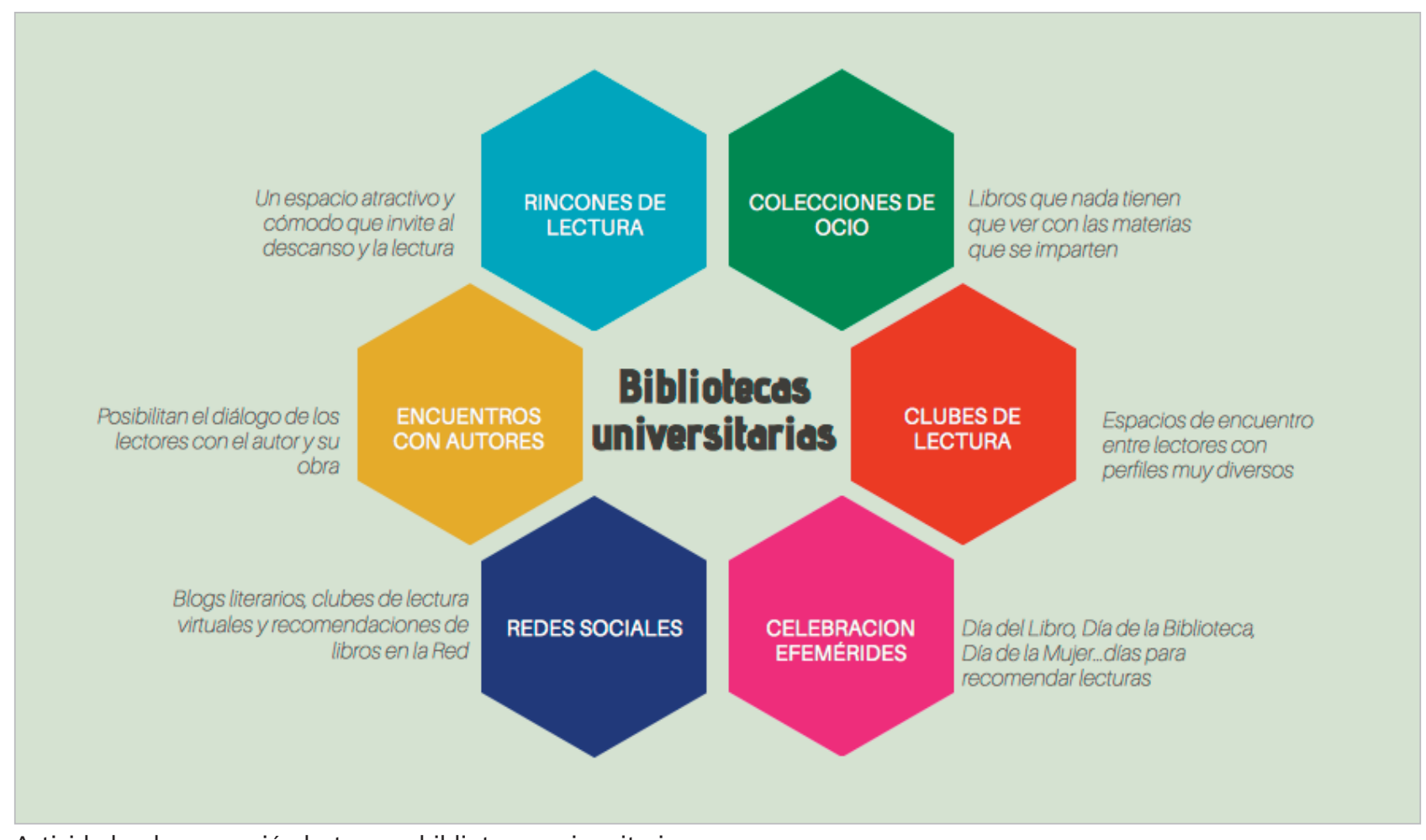

Actividades de promoción lectora en bibliotecas universitarias 
En los últimos años observamos una discreta tendencia en las bibliotecas universitarias por ofrecer servicios y actividades vinculadas con la promoción lectora a la comunidad universitaria en general y a los alumnos en particular. El primer paso para el fomento de la lectura en las bibliotecas universitarias ha consistido en dotarlas de colecciones denominadas de ocio, que buscan ofrecer a los usuarios libros para que lean por el simple placer de leer, sin necesidad de buscar en ellos otra finalidad más allá del entretenimiento. Como señalaban Penadés y Botías,

"nuestras colecciones, como biblioteca universitaria, están orientadas al aprendizaje, la investigación y la docencia en las áreas de conocimiento que se corresponden con las carreras y asignaturas" (Penadés; Botías, 2008, p. 109),

por lo que se trata de completar estas colecciones con otros fondos que poco o nada tienen que ver con las materias de estudio que se imparten en el campus. Surge así la necesidad de completar las colecciones con libros de lectura atractivos, centrados en temas actuales y de géneros diversos, entre los que la literatura de calidad debe tener un lugar destacado. El objetivo de estas nuevas colecciones es ofrecer a la comunidad universitaria una oferta variada y atractiva de lecturas que hasta ahora no se encontraba en estas bibliotecas, intentando promocionar la lectura recreativa como complemento a la lectura académica y formativa.

Una vez creadas estas colecciones se ha buscado promocionarlas y acercarlas a los usuarios de distintas formas, generalizándose la creación de espacios para la lectura. Además de dar visibilidad a estas obras, se busca crear un ambiente atractivo y cómodo que invite al descanso y la lectura. En estos espacios, junto a un mobiliario acogedor y confortable que invita al descanso y el esparcimiento, el usuario puede encontrar una oferta variada de lecturas con las que cubrir sus ratos libres: prensa diaria, revistas de divulgación, una selección de obras literarias y libros de distintos temas que se van renovando periódicamente para ofrecer una muestra significativa de las posibilidades temáticas que incluyen las colecciones de ocio. En definitiva, se trata de mostrar un escaparate atractivo que capte la atención de los usuarios, pero sobre todo les acerque a los libros, potenciando de forma especial la lectura de obras literarias, por la importancia que estas tienen para el desarrollo y mantenimiento del hábito lector (Sánchez-García; Santos-Recuenco, 2012, p. 50).

La organización de clubes de lectura ha sido una de las primeras iniciativas en cuanto a promoción lectora que se comenzó a poner en marcha en las bibliotecas universitarias, siendo actualmente una de las actividades más consolidadas. La importancia de los clubes de lectura en el ámbito universitario radica, principalmente, en su definición como un espacio integrador en el que entran en contacto, en torno a un mismo objetivo, distintas personas con edades, inquietudes, hábitos y gustos de lectura diferentes, siendo precisamente la diversidad de perfiles de los participantes uno de los elementos más enriquecedores de esta iniciativa (Sánchez-García; Yubero; Larrañaga, 2010). Un aspecto importante a señalar es la diversidad y heterogeneidad de sus participantes: la edad puede oscilar desde los 18 o 20 años hasta los 65 o 70 años. Como afirma Salcedo,

"esto, unido a los diferentes ámbitos de procedencia de los asistentes, nos lleva a ver la importancia de la diversidad y las relaciones intergeneracionales que se pueden dar en este tipo de clubes de lectura" (Salcedo, 2008, p. 92).

Además, el desarrollo de las redes sociales en las bibliotecas universitarias ha potenciado su uso para la creación de blogs literarios, clubes de lectura virtuales y recomendaciones de libros a partir de grupos y páginas de Facebook (Sánchez-García; Santos-Recuenco, 2014), entre otras iniciativas.

Una evidencia del papel que va adquiriendo la promoción de la lectura en las bibliotecas universitarias españolas es la reciente publicación del informe Promoción de la lectura en bibliotecas universitarias, desde la Línea Estratégica 1 de Rebiun (2018). Este estudio, basado en una encuesta dirigida a los directores/as de las bibliotecas que integran la red, se hace eco por primera vez de este tipo de actividades en el contexto universitario. Entre los resultados que arroja el informe, observamos cómo todavía se trata de una actividad minoritaria si tenemos en cuenta la información de las bibliotecas participantes: de las 75 bibliotecas que integran la red sólo 39 contestaron al estudio; de ellas 3 señalaron no ofrecer este tipo de actividades. Las 36 restantes no contestaron a la encuesta, lo que nos lleva a suponer que muchas de las bibliotecas que optaron por no participar en el estudio lo hacen precisamente por no ofrecer este tipo de actividades. En cuanto al tipo de actividades el informe señala la celebración del Día del libro como la más extendida ( 35 bibliotecas) y después un abanico de actividades que incluyen:

- oferta de colecciones de ocio y espacios de lectura (24 bibliotecas);

- presentaciones de libros (16 bibliotecas);

- concursos literarios (16 bibliotecas);

- encuentros con autores (14 bibliotecas); 
- campañas de promoción de la lectura y escritura (lo señalan 17 bibliotecas, pero sin especificar en qué se concretan esas campañas).

Sorprende que dentro de las actividades referidas en el estudio, no se ofrezca el dato específico de la oferta de clubes de lectura, siendo una de las actividades por excelencia dentro de los programas de promoción lectora y una actividad consolidada en muchas bibliotecas universitarias (Sánchez-García; Yubero; Larrañaga, 2010). En líneas generales las bibliotecas participantes en la encuesta declaran tener un impacto bajo sobre la comunidad universitaria, resultando complicado llegar a los alumnos, dato del que se hacen eco otros estudios.

Los resultados que arroja este informe nos muestran cómo aún queda mucho camino por recorrer para acercar la lectura a los jóvenes universitarios, más aún si tenemos en cuenta que, como señalan Sánchez-García y Yubero (2015), se trata de experiencias puntuales, no siendo habitual que estas acciones estén reflejadas dentro de los planes estratégicos ni del servicio ni de la propia institución. Además, es habitual que las bibliotecas se encuentren con diferentes barreras a la hora de poner en marcha estos servicios, siendo la falta de presupuesto y de personal los problemas comunes y recurrentes en la mayoría.

Finalmente, señalar la importancia de evaluar los resultados directos que este tipo de actuaciones tienen sobre los estudiantes. Muchos de los estudios sobre promoción de la lectura se centran en evaluar los procesos en sí mismos, valorando la eficacia de los métodos en relación con datos tan simples como el número de participantes y su satisfacción en las acciones, pero sin llegar a profundizar en el objetivo de todas estas acciones: la creación de lectores competentes y la consolidación del hábito lector.

¿Realmente por organizar actividades en torno al Día del libro fomentamos la lectura? ¿Aumenta la disponibilidad de colecciones de ocio el gusto lector de los alumnos? ¿Quién participa realmente en los clubes de lectura universitarios y en los encuentros con autores? ¿Son efectivas las recomendaciones y selecciones de lecturas que realizamos? Son demasiados interrogantes a los que muchas de las actividades que venimos realizando no pueden

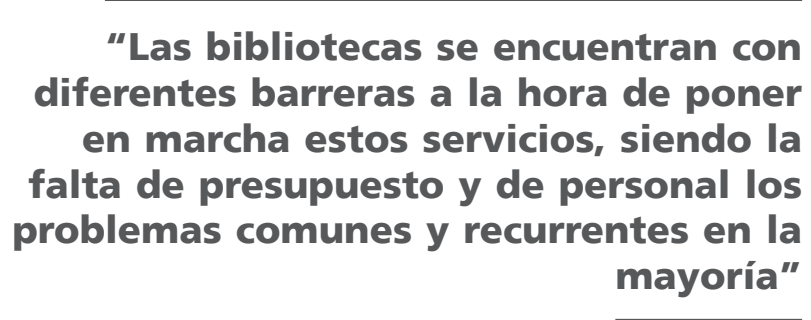
responder.

La promoción de la lectura en el ámbito universitario es una tarea compleja y exigente que debemos asumir, pero haciéndolo desde el compromiso que supone formar estudiantes competentes y críticos, en una sociedad en la que la lectura se convierte en un acto cada vez más complejo y necesario.

\section{Referencias}

Carlino, Paula (2013). "Alfabetización académica 10 años después". Revista mexicana de investigación educativa, v. 18, n. 57 , pp. 355-381. http://www.redalyc.org/pdf/140/14025774003.pdf

Cunningham, Anne E.; Stanovich, Keith E. (1991). "Tracking the unique effects of print exposure in children: Association with vocabulary, general knowledge, and spelling". Journal of educational psychology, v. 83, n. 2, pp. 264-274.

https://doi.org/10.1037/0022-0663.83.2.264

Dezcallar, Teresa; Clariana, Mercèe; Cladellas, Ramón; Badia, Mar; Gotzens, Concepción (2014). “La lectura por placer: su incidencia en el rendimiento académico, las horas de televisión y las horas de videojuegos". Ocnos, n. 12, pp. 107-116.

https://doi.org/10.18239/ocnos_2014.12.05

Elche, María; Sánchez-García, Sandra; Yubero, Santiago (2019). "Lectura, ocio y rendimiento académico en estudiantes universitarios del área socioeducativa". Educación XX1, v. 22, n. 1, pp. 215-237.

https://doi.org/10.5944/educxx1.21548

Elliott, Julie (2009). "Barriers to extracurricular reading promotion in academic libraries". Reference and user services quarterly, v. 48, n. 4, pp. 340-346.

https://doi.org/10.5860/rusq.48n4.340

Fiz, María-Reyes; Goicoechea, María-Jesús; Ibiricu, Olga; Olea, María-José (2000). "La comprensión lectora y su repercusión en el rendimiento escolar". Huarte de San Juan. Psicología y pedagogía, n. 6-7, pp. 75-108.

Fraguela-Vale, Raúl; Pose-Porto, Héctor; Varela-Garrote, Lara (2016). "Tiempos escolares y lectura". Ocnos, v. 15, n. 2, pp. 67-76.

https://doi.org/10.18239/ocnos_2016.15.2.1099 
Gambrell, Linda B. (2015). "Getting students hooked on the reading habit". Reading teacher, v. 69, n. 3, pp. $259-263$. https://doi.org/10.1002/trtr.1423

Gil-Flores, Javier (2011). “Hábitos lectores y competencias básicas en el alumnado de educación secundaria obligatoria". Educación XX1, v. 14, n. 1, pp. 117-134.

https://doi.org/10.5944/educxx1.14.1.274

Gilbert, Julie; Fister, Barbara (2011). "Reading, risk and reality: College students and reading for pleasure". College \& research libraries, v. 72, n. 5, pp. 474-495.

https://doi.org/10.5860/crl-148

Gladwin, Rachel; Goulding, Anne (2012). "Recreational reading in universities libraries in the United Kingdom". New review of academic librariannship, v. 18, n. 2, pp. 140-164.

https://doi.org/10.1080/13614533.2012.709213

González, María-José; Delgado, Miriam (2009). "Rendimiento académico y enseñanza-aprendizaje de la lectoescritura en Educación Infantil y Primaria: un estudio longitudinal". Infancia y aprendizaje, v. 32, n. 3, pp. 265-276. https://doi.org/10.1174/021037009788964114

González-Anleo, Juan-María; López-Ruiz, José A. (2017). Jóvenes españoles entre dos siglos 1984-2017. Madrid: Fundación SM. Observatorio de la Juventud en Iberoamérica.

https://drive.google.com/file/d/1AAwhVJIzEhoicuRM4It9ducBP3fSEJfo/view

Howard, Vivian (2011). "The importance of pleasure reading in the lives of young teens: Self-identification, self-construction and self-awareness". Journal of librarianship and information Science, v. 43, n. 1, pp. 46-55. https://doi.org/10.1177/0961000610390992

Hughes-Hassell, Sandra; Rodge, Pradnya (2007). "The leisure reading habits of urban adolescents". Journal of adolescent $\&$ adult literacy, v. 51, n. 1, pp. 22-33.

https://doi.org/10.1598/JAAL.51.1.3

Larrañaga, Elisa; Yubero, Santiago (2005). “El hábito lector como actitud. El origen de la categoría de falsos lectores". Ocnos, n. 1, pp. 43-60.

https://doi.org/10.18239/ocnos_2005.01.04

Larrañaga, Elisa; Yubero, Santiago; Cerrillo, Pedro C. (2008). Estudio sobre los hábitos de lectura de los universitarios españoles. Madrid: Cepli/SM. ISBN: 9788467524390

Lee, Jihyun (2014). "Universal factors of student achievement in high-performing Eastern and Western countries". Journal of educational psychology, v. 106, n. 2, pp. 364-374.

https://doi.org/10.1037/a0035609

Lluch, Gemma; Sánchez-García, Sandra (2017). "La promoción de la lectura: un análisis crítico de los artículos de investigación". Revista española de documentación científica, v. 40, n. 4.

https://doi.org/10.3989/redc.2017.4.1450

Mol, Suzanne E.; Jolles, Jelle (2014). "Reading enjoyment amongst non-leisure readers can affect achievement in secondary school". Frontiers in psychology, n. 5.

https://doi.org/10.3389/fpsyg.2014.01214

Molina-Villaseñor, Leandro (2006). “Lectura y educación: los hábitos lectores y su repercusión académica en la ESO". Ocnos, n. 2, pp. 103-120.

https://doi.org/10.18239/ocnos_2006.02.07

Moyano, Estela-Inés (2004). "La escritura académica: una tarea interdisciplinaria a lo largo del currículo universitario". Revista textura, v. 4, n. 4, pp. 109-120.

https://doi.org/10.14409/texturas.v1i4.2824

Pascual-Gómez, Isabel; Carril-Martínez, Isabel (2017). “Relación entre la comprensión lectora, la ortografía y el rendimiento: un estudio en Educación Primaria". Ocnos, v. 16, n. 1, pp. 7-17.

https://doi.org/10.18239/ocnos_2017.16.1.1167

Penadés, Honorio; Botias, Rocio (2008). "La biblioteca universitaria que quería ser una biblioteca pública". Educación y biblioteca, v. 20, n. 165, pp. 109-116.

Rebiun (2018). Promoción de la lectura en bibliotecas universitarias. Rebiun Línea 1. http://hdl.handle.net/20.500.11967/252

Ritchie, Stuart J.; Bates, Timothy C. (2013). "Enduring links from childhood mathematics and reading achievement to adult socioeconomic status". Psychological science, v. 24, n. 7, pp. 1301-1308.

https://doi.org/10.1177/0956797612466268

Salcedo, Ángel (2008). "Club de Lectura Universidad de Murcia: un recorrido por la lectura en sus seis años de vida". Educación y biblioteca, v. 20, n. 165, pp. 89-93.

http://hdl.handle.net/10366/119527 
Sánchez-García, Sandra; Santos-Recuenco, Eloísa (2012). "Leer por leer. La promoción de la lectura desde la Biblioteca Universitaria". Mi biblioteca, n. 28, pp. 48-51.

Sánchez-García, Sandra; Santos-Recuenco, Eloísa (2014). “Biblioteca 2.0 y alfabetización lectora: una visión crítica". En: Yubero, Santiago; Larrañaga, Elisa (eds.). Propuestas socioeducativas para la alfabetización lectora. Cuenca: Universidad de Castilla-La Mancha, pp. 33-42. ISBN: 9788490441039

Sánchez-García, Sandra; Yubero, Santiago (2015). “Leer en la universidad: la promoción de la lectura desde la biblioteca universitaria". En: Sánchez-García, Sandra; Yubero, Santiago (coords.). Las bibliotecas en la formación del hábito lector. Cuenca: Servicio Publicaciones UCLM, pp. 159-188. ISBN: 9788490441329

Sánchez-García, Sandra; Yubero, Santiago (2017). "Promover la lectura en las bibliotecas: estrategias y perspectivas". Lieratura em debate, v. 11, n. 11, pp. 146-161.

http://revistas.fw.uri.br/index.php/literaturaemdebate/article/view/2710

Sánchez-García, Sandra; Yubero, Santiago; Larrañaga, Elisa (2010). "El papel de la biblioteca universitaria en la formación del hábito lector". En: Bibliotecas: rompiendo barreras, tejiendo redes. Málaga: Asociación Andaluza de Bibliotecarios.

http://hdl.handle.net/10578/93

Yubero, Santiago; Larrañaga, Elisa (2015). "Lectura y universidad: hábitos lectores de los estudiantes universitarios de España y Portugal". El profesional de la información, v. 24, n. 6, pp. 713-719.

https://doi.org/10.3145/epi.2015.nov.03

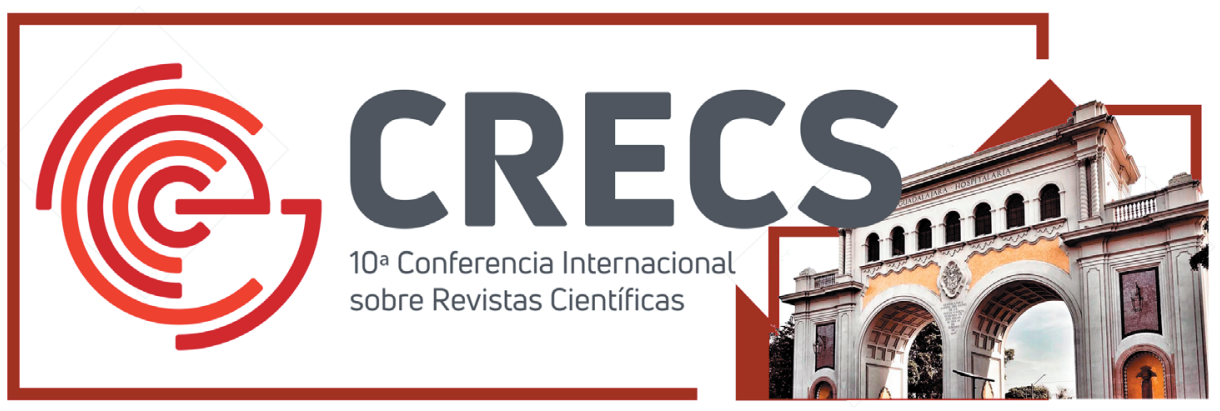

23 - 24 DE OCTUBRE UNIVERSIDAD PANAMERICANA

\section{0a Conferencia internacional sobre revistas científicas (CRECS) 2019}

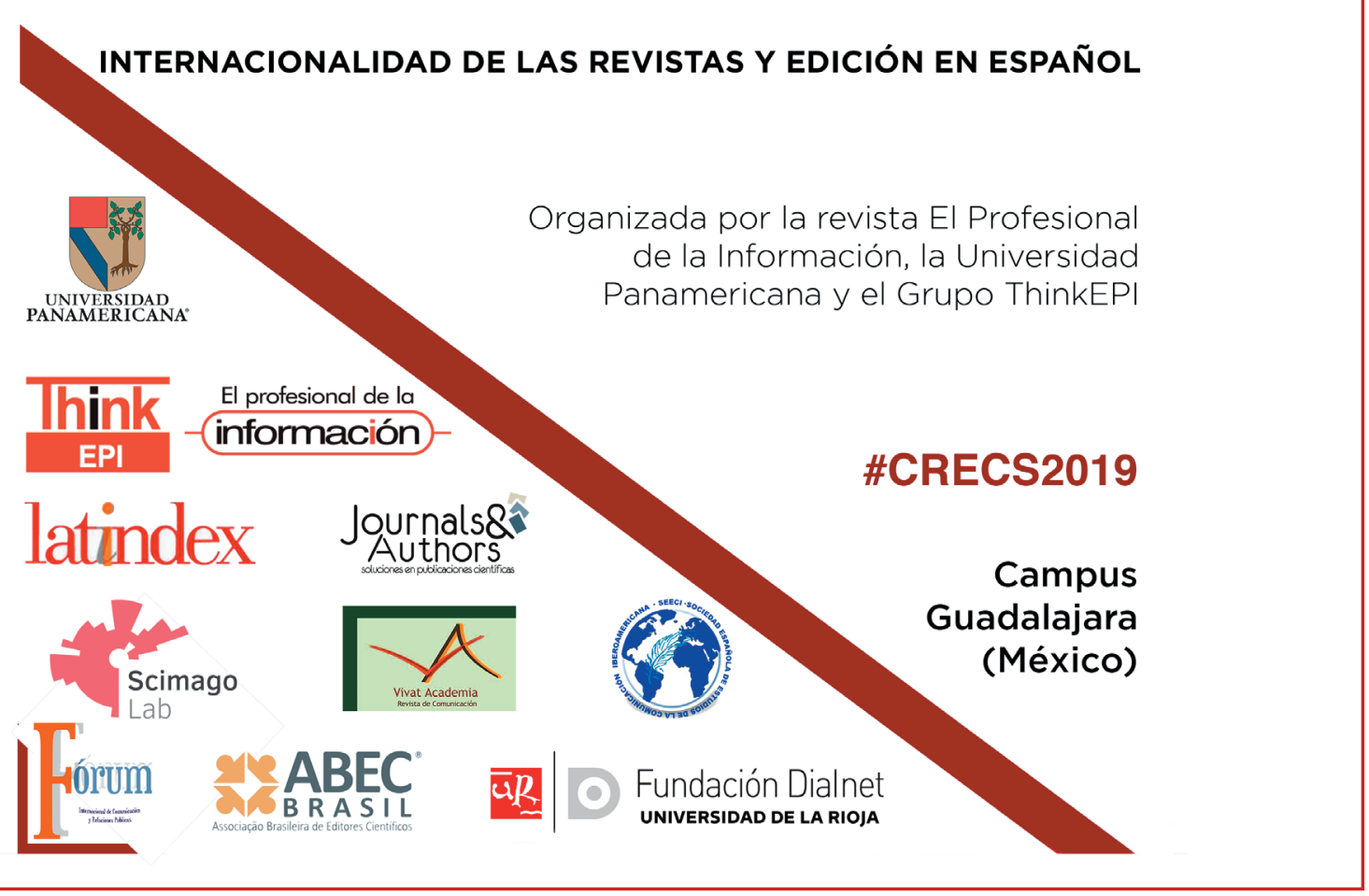

